\title{
Improving measles syndromic surveillance via dried blood spot testing in Laos 2016-17
}

\author{
Andrew D. Nguyen, Andrew Dang Khai Nguyen, Chanthavy Soulaphy, Michael Marks, Jennie \\ Musto
}

Queensland Health, Australia

Objective

To evaluate whether dried blood spot (DBS) testing improves diagnostic uptake in Vientiane Capital City province, Lao People's Democratic Republic (PDR) compared to conventional diagnostic techniques (venous blood by venepuncture) during syndromic surveillance from 2016-17. To also explore reasons for low blood sampling uptake via quantitative results and qualitative responses from health care workers; in addition to the perceived acceptance of DBS compared to venepuncture.

\section{Introduction}

The Lao PDR is aiming for measles elimination despite ongoing outbreaks of the disease. Outbreak detection in the country relies on recognising cases meeting a set "fever and rash" case definition incorporated into the syndromic surveillance system run by the National Center for Laboratory and Epidemiology (NCLE). Suspected cases are passively identified by presentations at health care facilities, with information forwarded to the NCLE's Early Alert and Response Network (EWARN) along with event-based reported data [1]. World Health Organization (WHO) measles surveillance guidelines require $\geq 80 \%$ of "fever and rash" cases be sampled for testing; currently only $20 \%$ sampling occurs in Laos [2,3]. Sampling using DBS has been proposed as an alternative to conventional venepuncture in facilitating suspected measles case detection. In this study, DBS was proposed to improve blood uptake of syndromic cases, by evaluating whether it increased ascertainment compared to conventional venepuncture. It also analysed reasons for poor diagnostic uptake among healthcare personnel involved in syndromic surveillance.

\section{Methods}

A mixed methods study involving a cross-sectional study and a qualitative survey was undertaken in Vientiane Capital City Province. Nine district and central hospitals were included to represent the general provincial population composition. Surveillance data were provided through routine surveillance staff review of hospital logbooks and community health centres together with case investigation forms forwarded to the NCLE's EWARN. A sample size of 166 was calculated with $80 \%$ power to detect a $20 \%$ difference in uptake in syndromic surveillance between DBS and venepuncture. A 1:1 matching of venepuncture and DBS notifications was set. A randomly selected sample of 105 from a total of 204 notifications of "fever and rash" from June-September 2016 during routine venepuncture-based surveillance was compared with a preliminary 13 collected notifications from a proposed 100 in June-September 2017 following introduction of DBS to routine use. Resource limitations in 2017 restricted the DBS samples $(\mathrm{n}=13)$ analysed at this preliminary stage.

Reasons for baseline poor sampling uptake using 2016 venepuncture data $(n=204)$ were separately explored according to categories including demographics, hospital, provisional diagnosis and measles immunisation. Microsoft Excel 2007 and STATA v14.0 were used for descriptive, univariate and multivariate analyses of explanatory variables. Qualitative questionnaires were physically administered to personnel at each hospital according to their involvement in syndromic surveillance in July-September 2016-17. Given time constraints, a limited sample of surveillance personnel involved in the study $(n=7)$ completed qualitative questionnaires. Questionnaires explored reasons for poor uptake using a framework analysis of five themes focused on demographics, aetiology of reasoning, venous and DBS acceptance, and sampling preference. Patterns were correlated with quantitative data.

\section{Results}

Baseline characteristics were similar across both study periods. A high frequency of "fever and rash" cases was detected among those $0-9$ years $(71.19 \%)$ in the study periods analysed. Blood samples were obtained from $25.77 \%$ of "fever and rash" notifications using conventional venepuncture, reflecting current poor diagnostic uptake. Direct comparison of 2016 and 2017 periods was underpowered at the time of analysis $(n=105$ vs $n=13)$. But preliminary results indicated DBS had no difference in improving diagnostic uptake (23.07\% vs 25.77\%; OR 0.83; CI 0.14-3.41) compared to baseline venepuncture. Exploration of baseline 2016 venepuncture data $(n=204)$ revealed only three "fever and rash" notifications were forwarded to the EWARN from hospitals involved in this study period. Hospitals also varied in blood sampling. Presenting at Nasaithong district hospital was less associated

SDS Annual Conference Proceedings 2019. This is an Open Access article distributed under the terms of the Creative Commons AttributionNoncommercial 4.0 Unported License (http://creativecommons.org/licenses/by-nc/3.0/), permitting all non-commercial use, distribution, and reproduction in any medium, provided the original work is properly cited. 
with uptake than not presenting there (OR 0.15; CI 0.003-0.99). Those presenting at Xaythany district (OR 4.53; CI 1.66-12.56) and Settathirath central (OR 3.09; CI 1.39-6.77) hospitals had greater odds of blood sampling than those who did not. Logistic regression indicated a borderline increased odds of 1.02 (CI 1.00-1.05) for having bloods taken with each year of increased age. With provisional diagnoses, there were six suspected measles cases, with only three suspected cases being tested. Measles diagnosis was not associated with blood uptake (OR 2.35; CI 0.69-7.55). Varicella diagnosis was less associated with uptake than not having varicella (OR 0.06; CI 0.001-0.39), even after multivariable analysis.

Qualitative results described staff concerned with competing demands from clinical workloads and fulfilling syndromic surveillance reporting requirements. A common theme was in neglecting the syndromic case definition in lieu of the clinical case definition, fueling misunderstanding of reporting requirements. Patient cultural beliefs were identified as being associated with altered blood sampling rates. Respondents were equally split on patient preference between DBS and venepuncture techniques.

\section{Conclusions}

Results for DBS and venepuncture analysis were limited by data collection. However, this is one of the first studies to analyse the use of DBS in syndromic surveillance. Preliminary results revealed no difference in diagnostic uptake between DBS and venepuncture, indicating poor blood ascertainment regardless of technique. Collected data reflected current diagnostic uptake trends in the Lao PDR and was representative of Vientiane Capital City Province. Quantitative and qualitative analyses of uptake indicate weaknesses in syndromic surveillance, varying by institution, cultural beliefs and understanding of case definitions. Completion of DBS data collection will be expected to corroborate current findings. Further studies exploring diagnostic uptake limitations and DBS viability in low resource settings may build on this data and inform syndromic surveillance opinion on using DBS.

\section{Acknowledgement}

This project was supported by the NCLE (Lao PDR), the Chadwick Trust (UK) and the London School of Hygiene \& Tropical Medicine. Supervision was assisted by WHO Lao PDR Country Office personnel.

\section{References}

1. Sengkeopraseuth B. et al. 2016. Hidden varicella outbreak, Luang Prabang Province, the Lao People's Democratic Republic, December 2014 to January 2015. Western Pac Surveill Response J. 7(1), 1-5. PubMed https://doi.org/10.5365/wpsar.2015.6.2.010

2. World Health Organization. WHO-recommended standards for surveillance of selected vaccine-preventable diseases. In: Department of Vaccines and Biologicals. Geneva, Switzerland: World Health Organization. 2003; 1-59.

3. Musto J. Measles epidemiology in Lao People's Democratic Republic. (Unpublished data). 2017.

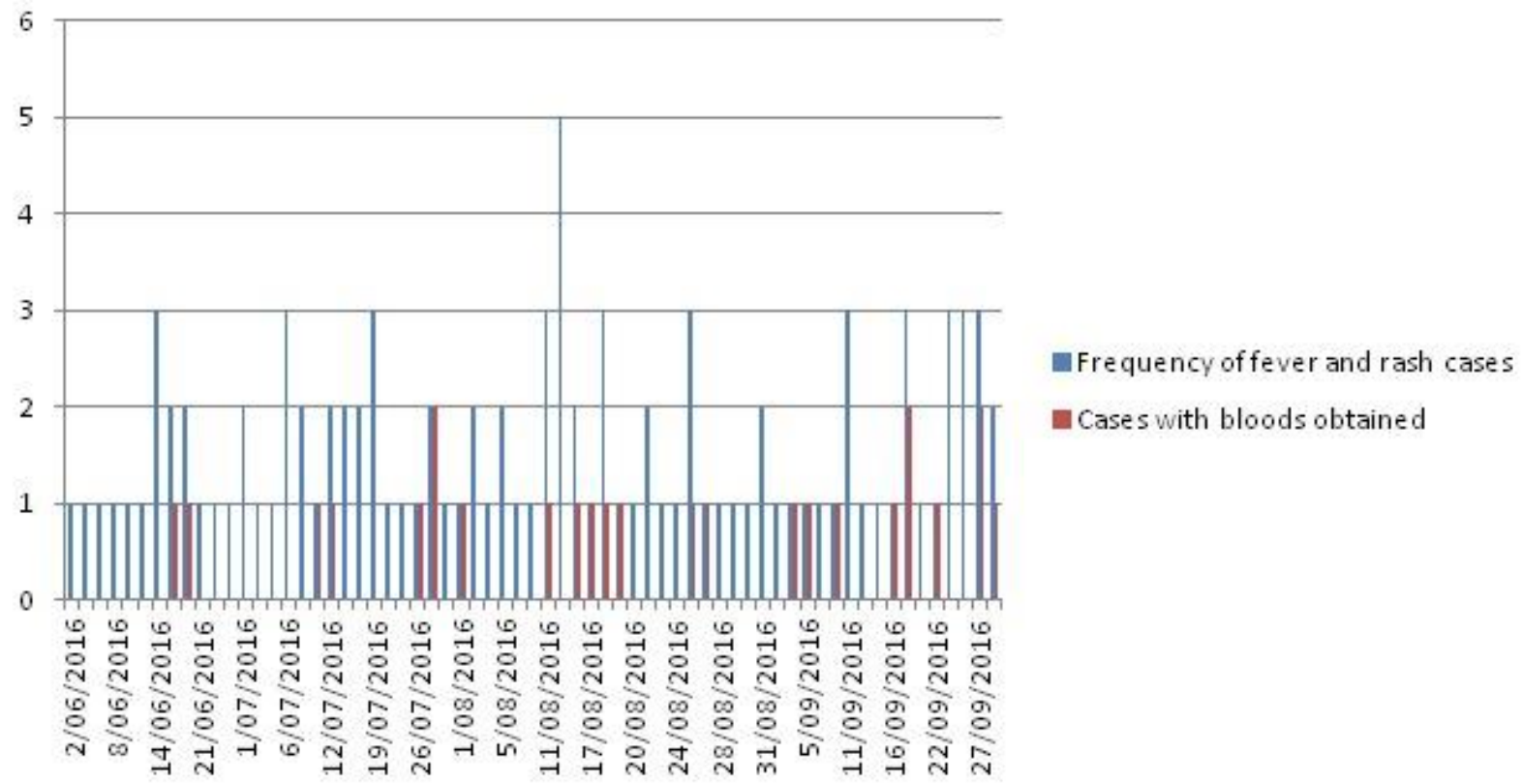

